\title{
LOW-FREQUENCY VARIABILITY IN THE BLOOD VOLUME AND IN THE BLOOD VOLUME Pulse Measured By Photoplethysmography
}

\author{
Meir Nitzan, ${ }^{\dagger}$ Sergei Turivnenko,${ }^{\dagger}$ Adina Milston, ${ }^{\dagger}$ Anatoly Babchenko, ${ }^{\dagger}$ \\ and Yonah Mahler \\ Jerusalem College of Technology, Department of Electro-Optics and Applied Physics, P.O. \\ Box 16031, Jerusalem 91160, Israel; ${ }^{\ddagger}$ Hadassah University Hospital, Medical \\ Engineering Division, Jerusalem, Israel \\ (Paper JBO-016 received June 14, 1995; revised manuscript received Dec. 27, 1995; accepted for publication Dec. 27, 1995)
}

\begin{abstract}
Besides heart rate and arterial blood pressure, several parameters of the cardiovascular system fluctuate spontaneously. In the current study, the fluctuations of tissue blood content and blood volume pulse were investigated using two parameters of the photoplethysmographic (PPG) signal: the parameter BV, defined by: $\mathrm{BV}=$ Const $-\mathrm{BL}$ where BL is the baseline of the PPG signal, and the amplitude (AM), which are related to the blood volume and to the systolic blood volume increase, respectively. The PPG measurements were performed on the fingertips of ten healthy male subjects for 5 to $10 \mathrm{~min}$ and the PPG signal was digitally analyzed. Both BV and AM show low frequency fluctuations, which, for 23 out of 26 examinations, were positively correlated, with a lag of BV relative to AM. In three examinations, however, the two parameters were inversely correlated. A lower correlation was found between each of these parameters and the PPG period, which is actually the cardiac period. The results show that several mechanisms are involved in the spontaneous periodic fluctuations in the vascoconstriction level, which are known to be mediated by the sympathetic nervous system. The digital PPG provides, therefore, a potential tool for evaluating the role of the sympathetic nerves in the regulation of the microcirculation.
\end{abstract}

Keywords photoplethysmography; heart rate variability; tissue blood volume; blood volume pulse; sympathetic nervous system.

\section{BACKGROUND}

\subsection{SPONTANEOUS FLUCTUATIONS IN THE CARDIOVASCULAR SYSTEM}

Various parameters of the cardiovascular system show spontaneous oscillations in well-defined frequencies. Heart rate variability (HRV), which is the spontaneous fluctuation of the heart rate (or the cardiac cycle period) around its mean value, is associated with activity of the autonomic nervous system. The power spectrum of HRV is mainly composed of three frequency ranges: low and medium frequencies (below $0.05 \mathrm{~Hz}$ and at about 0.1 $\mathrm{Hz}$, respectively) which are attributed to the activity of the sympathetic and the parasympathetic nervous systems, and high frequency (around $0.3 \mathrm{~Hz}$ ), which is caused by the influence of respiration on the cardiovascular system via the parasympathetic nervous system. ${ }^{1-5}$ Similar oscillations are also obtained in arterial blood pressure measurements, both during the respiratory period and during longer periods. ${ }^{1,2,5}$

Address all correspondence to Meir Nitzan. E-mail:nitzan@horaah.jct.ac.il
Spontaneous fluctuations were also found for other parameters of the peripheral blood circulation system. Burton ${ }^{6}$ used air plethysmography to continuously measure the blood content in the fingertip and its blood volume systolic pulse and found low-frequency fluctuations in these parameters which were proved to be related to the sympathetic nervous activity. In a recent study, ultrasound Doppler velocity was measured in the brachial artery simultaneously with continuous measurement of arterial blood pressure in the finger, showing an inverse correlation between the two parameters. Oscillations were also found in the average value of the photoplethysmographic (PPG) signal, and in that of laser Doppler flowmetry, showing correlation between the two signals, which are related to tissue blood volume and tissue blood flow, respectively. ${ }^{8}$ In another study, ${ }^{9}$ the fluctuations in tissue blood flow measured by laser Doppler flowmetry were compared with those of the pulsatile PPG signal, and a positive correlation was found between the two parameters. 


\subsection{PHOTOPLETHYSMOGRAPHY}

The PPG signal has two components: the baseline of the PPG pulse and the pulse itself, sometimes called the dc and the ac components, respectively. The baseline of the PPG signal is inversely related to the blood volume in the tissue under examination, since higher blood volume results in higher absorption of the light. The pulsatile component of the PPG signal mainly depends on the tissue blood volume increase during systole,, 10 which is determined by the difference between the amounts of blood flowing into the tissue and out of it during systole. During systole, when blood flows into the tissue, a portion of tissue blood volume is drained from the tissue via the venous system, and that portion depends on the compliance and the resistance to flow of the blood vessels. ${ }^{11,12}$ The systolic increase in tissue blood volume depends, therefore, on the tissue blood flow waveform (which depends on the local arterial blood pressure waveform) and on the vascular compliance and resistance. The PPG pulsatile amplitude also depends on the tissue blood content (in addition to its effect on the vascular compliance) since the light absorption by the blood results in attenuation of the PPG pulse.

In a previous study, ${ }^{13,14}$ the PPG signal in the fingertip was measured and three parameters were digitally derived for each pulse: its baseline, its amplitude, and the cardiac cycle period. The power spectrum of the spontaneous fluctuations in these three parameters showed significant low-frequency fluctuations for the baseline and amplitude curves. Fluctuations in medium and high frequency also appeared for these curves, but they were smaller than the corresponding fluctuations in the cardiac cycle period. In the present study, the lowfrequency fluctuations of the baseline and amplitude of the PPG signal and its period were examined in the time domain, and the correlations between them were investigated.

\section{MATERIALS AND METHODS}

Reflection photoplethysmographic examinations were performed on the palmar surface of the index fingers of 10 male subjects aged 21 to 64 years. The subjects were seated during the examination, which lasted for 5 to $10 \mathrm{~min}$, with their hands comfortably laid on a table, at heart level. Two to four examinations were performed on each subject. Room temperature was 21 to $24^{\circ} \mathrm{C}$.

The PPG probe consisted of a light-emitting diode (LED) of $840 \mathrm{~nm}$ (OP295, Optec) as a light source, and a photodetector (S1223-01, Hamamatsu), located $13 \mathrm{~mm}$ apart. For this distance between the light source and the photodetector, the amplitude of the PPG pulse was 2 to $5 \%$ of the average light measured by the detector. The PPG signal from the detector was amplified, sampled by an A/D card (100 samples per second), and the data stored in the computer memory. After the examina-

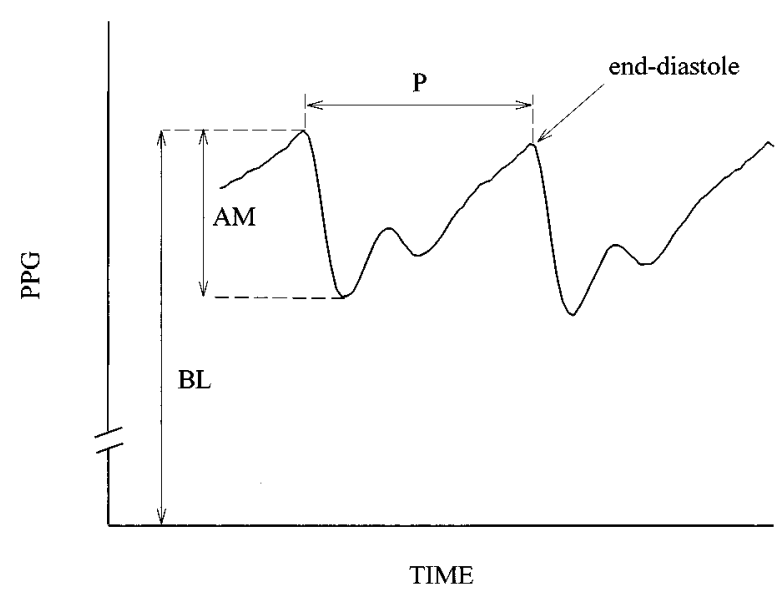

Fig. 1 Typical PPG signal. BL, baseline of the pulse; $A M$, its amplitude; $P$, its period.

tion, the data was automatically analyzed for the detection of the end-diastolic maximum and the systolic minimum in each PPG pulse signal. Then, for each pulse, the three parameters shown in Figure 1, the baseline of the curve (BL), its amplitude $(\mathrm{AM})$, and period $(\mathrm{P})$ were determined. Note that the PPG signal is not inverted (as is usually done when the PPG curve is presented) and decreases during systole, according to the intensity of the scattered light from the tissue. The baseline of the curve, BL, is inversely related to the blood volume in the tissue, so that qualitative assessment of the latter can be provided by the parameter BV, given by: $\mathrm{BV}=$ Const $-\mathrm{BL}$, where Const is some arbitrary constant chosen so that BV values are positive. Const can be arbitrary since only the fluctuations of BV were examined, while the absolute value of the baseline was used only in order to show that the PPG signal and its variability were small relative to the average baseline value.

This study was aimed at examining the lowfrequency fluctuations in the value of $\mathrm{BV}, \mathrm{AM}$, and $\mathrm{P}$ (which are of a 30-50 s period). Both lower and higher frequency fluctuations were filtered out. The relative high frequencies were reduced by using the smoothing method of the moving average, with linear decrease weights, of 15 adjacent points (each point being related to a single heart pulse). Fluctuations of very low frequency that occur as a result of physiological or emotional activity ${ }^{15}$ were reduced (trend removal) by performing, for each parameter, a 151-point moving average curve and subtracting it from the original curve. The averaging over 151 points was found to be effective for eliminating the very slow variations while minimally affecting the low-frequency fluctuations that were studied.

For four subjects, the PPG examination was performed simultaneously with ECG in order to check the reliability of the cardiac cycle period measurement by PPG. Both signals were then sampled (150 samples per second) and the ECG R-R intervals 


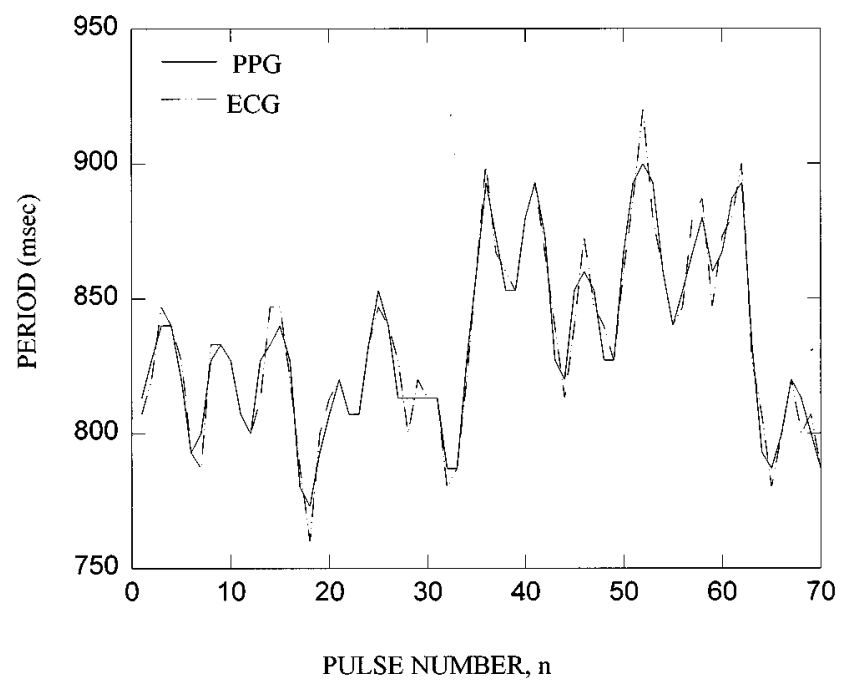

Fig. 2 Plot of simultaneous measurement of ECG R-R intervals and PPG pulse intervals as a function of time, for one of the subjects.

were compared with the PPG maximum-tomaximum intervals.

\section{RESULTS}

The simultaneous measurement of the cardiac cycle period by ECG and PPG provided similar results, as shown in Figure 2, which presents the raw data without smoothing and trend removal for one of the subjects. The correlation coefficient of the results obtained by the two methods was 0.98 to 0.99 , indicating that the PPG period variability actually represents the ECG R-R interval variability.

The PPG signal for all the subjects showed variability in its baseline, amplitude, and period. The PPG signal was 2 to $5 \%$ of the average baseline value, and typical relative fluctuations in its amplitude were 20 to $30 \%$ of the amplitude value. Typical baseline fluctuations were 3 to $5 \%$ of the average baseline. The average baseline was determined from the original pre-filtering data. Since the relative changes in the transmitted light are small, a linear relationship between the latter and the blood volume changes can be considered. The spontaneous fluctuations in the values of BV, AM, and P for one of the subjects are seen in Figure 3(a). Both high- and low-frequency variability appears in all the curves but the low-frequency fluctuations are more pronounced in the BV and AM curves while the high-frequency fluctuations dominate the $\mathrm{P}$ curve. The BV and AM curves show a high level of correlation which can be better seen after smoothing the curves and filtering out the high-frequency fluctuations. Figure 3(b) shows the curves of Fig. 3(a) after the smoothing of 15 adjacent points, which reduces the high- and medium-frequency fluctuations, and emphasizes the low-frequency ones.

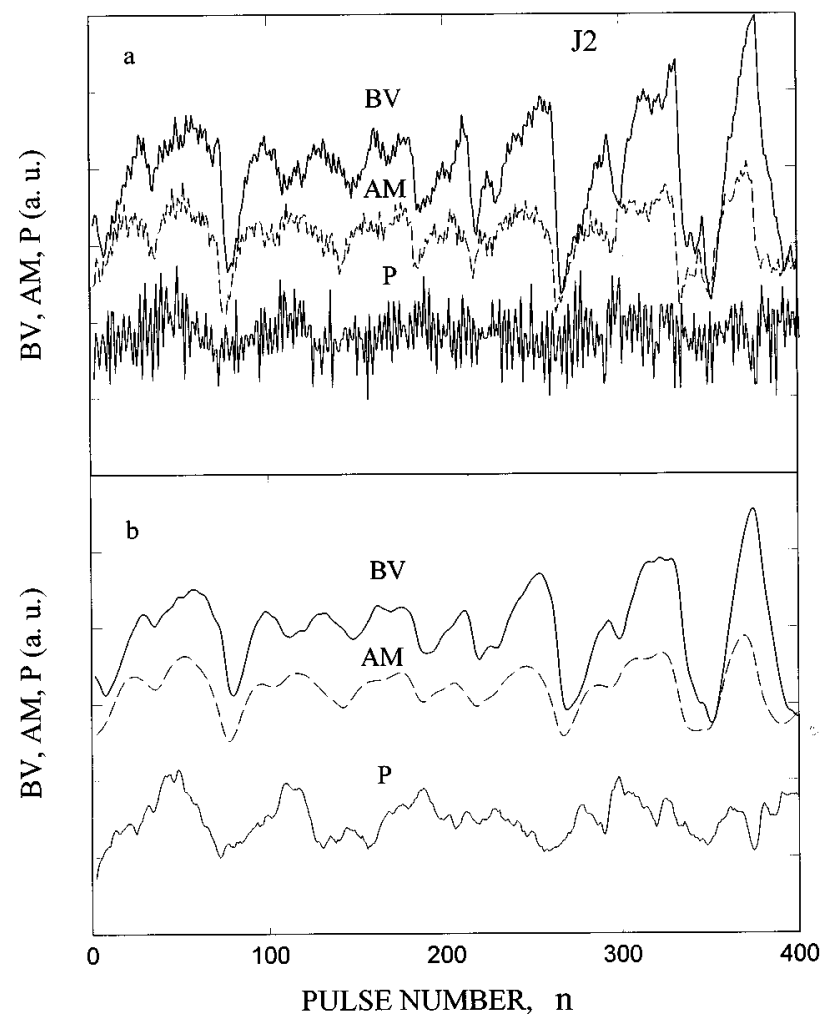

Fig. 3 The curves of $B V, A M$, and $P$ as a function of pulse number for one of the subjects, after trend removal, before (a) and after (b) smoothing. The high-frequency fluctuations in (a) are in the respiration rate.

The interdependence among the three parameters in the former examination is shown in Figure 4, which presents the cross-correlation function between each pair of the three parameters. The correlation coefficient for BV versus AM dependence is highest $(r=0.94)$ for the correlation between $\mathrm{BV}(n$ $+4)$ and $\operatorname{AM}(n)$. The correlation is lower, but statistically significant, for the BV and AM versus $P$ curves. However, in other examinations performed on the same subject, different correlation patterns were found. Figure 5 presents the results from another examination of the same subject as that in Fig. 3 which shows a negative correlation for BV versus AM curves, and Figure 6 presents an examination of the same subject in which different patterns of correlation can be found in the initial and final periods of the same examination. Under each set of curves, the cross-correlation function between BV versus $A M$ curves is presented [Figs. 5(b) and 6(b)].

The relationship between BV and AM could vary considerably among different examinations performed on the same subject, but for 23 out of the 26 examinations, the correlation coefficient was positive and for 17 of them the correlation coefficient was above 0.80 . In some examinations it could be seen that the low correlation coefficient originated due to short periods of negative correlation coefficient, as was shown in Fig. 6. In order to determine 


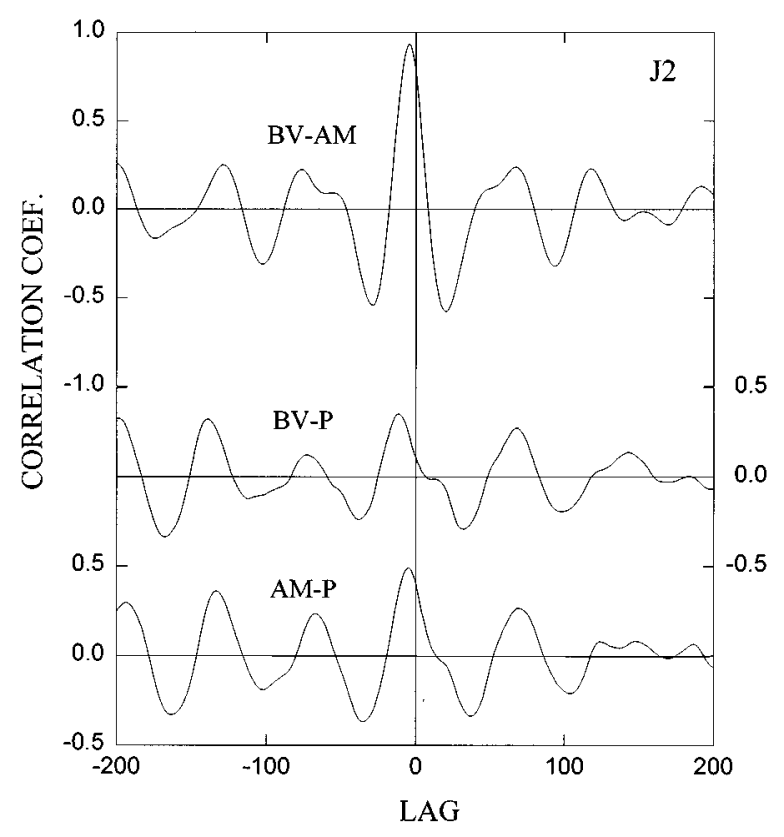

Fig. 4 The cross-correlation function for each pair of the three parameters-BV, $A M$, and $P$ for the examination presented in Fig. 3.

the lag between $\mathrm{AM}$ and BV curves, and in order to correlate $\mathrm{P}$ and $\mathrm{BV}$ and $\mathrm{AM}$, examinations of high positive correlation were selected. For each subject an (continuous) epoch of at least 300 pulses, in which the highest correlation coefficient of BV versus AM curves was found, was chosen, and for that epoch the correlation coefficients of the BV versus $A M, B V$ versus $P$, and $A M$ versus $P$ curves were determined and are presented in Table 1 . The table shows that all the subjects had epochs of high correlation between BV and AM (average maximum correlation coefficient is $0.92 \pm 0.03$ ), and that the BV curve lags behind the AM curve by 2 to 8 heartbeats. The slope of the BV versus AM curve is 3.14 to 5.06 , i.e., the $\mathrm{BV}$ fluctuations are 3 to 5 times higher than those of AM. In most of the other examinations performed on the same subjects (not shown in the table), the correlation coefficient of BV versus AM was positive (range 0.16 to 0.92 ), but for three examinations it was negative (range -0.62 to $-0.82)$.

The correlation between $\mathrm{P}$ and BV or AM, for the examinations shown in Table 1, is lower than the correlation between BV and AM. Table 1 shows the correlation coefficients between BV or AM and P with the corresponding lag. The last examination in the table is the only one that had a different sign for the two correlations, and in this examination the correlation coefficients have relatively low absolute value. For the other subjects both BV and AM showed either positive (6 subjects) or negative (3 subjects) correlations with $\mathrm{P}$, with an absolute correlation coefficient of 0.25 to 0.84 . For all the subjects, the correlation coefficient for AM versus $P$
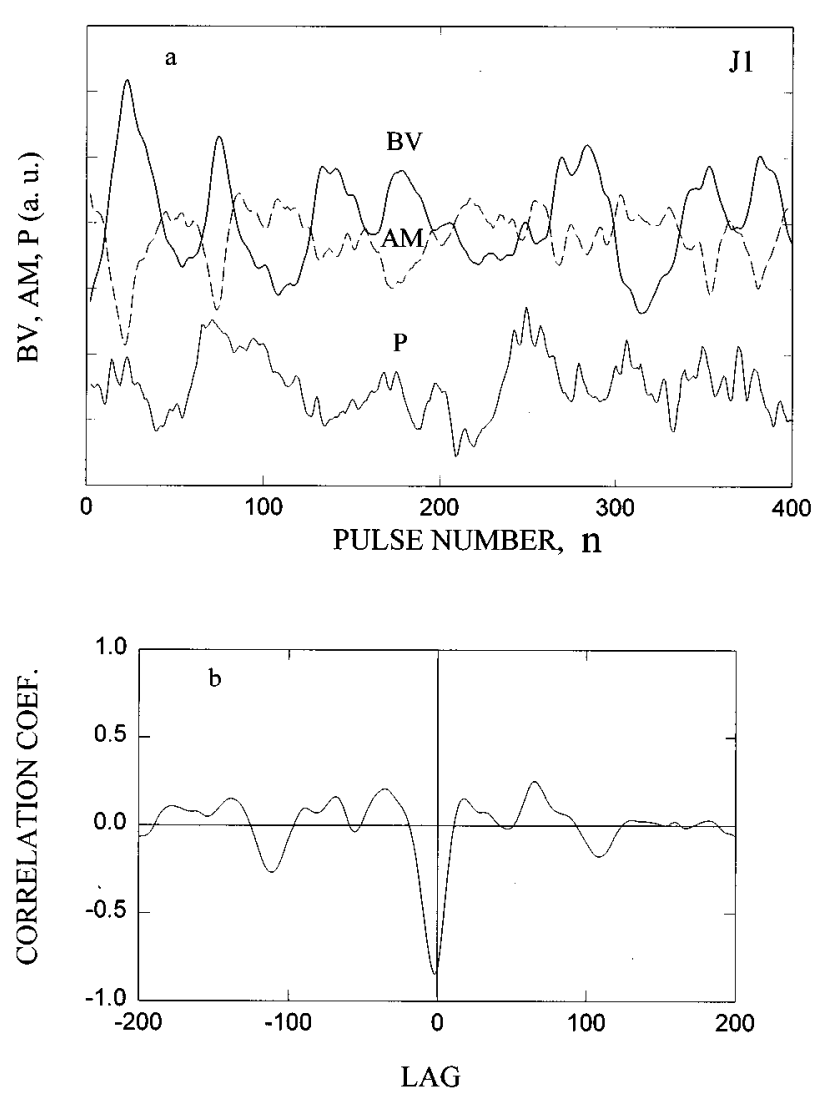

Fig. 5 (a) The curves of BV, $A M$, and $P$ as a function of pulse number after trend removal and smoothing for the same subject as in Fig. 3, in a different examination; (b) the cross-correlation function for BV versus $A M$ curves.

curves was higher than that for BV versus $P$ curves. It should be noted that even for subject 10 , the correlations with $\mathrm{P}$ are statistically significant $(P<0.01$ for $\mathrm{BV}$, and $P<0.005$ for $\mathrm{AM}$ ). For all other subjects, the correlation with $P$ has higher significance $(P$ $<0.0001)$.

\section{DiscUSSION}

In this study, photoplethysmography was used to evaluate low-frequency spontaneous fluctuations in tissue blood volume and the blood volume pulse in the fingertip. Similar low-frequency variability was found in heart rate and blood pressure measurements, and it was associated with fluctuations in the constriction level of the arterial system, mediated by the sympathetic nervous system. The lowfrequency fluctuations that appear in the heart rate are indirectly related to vasoconstriction variability, either because both are directly affected by sympathetic nervous activity or because an increase in the vasoconstriction level of blood vessels increases blood pressure, which reduces sympathetic nervous activity through a negative feedback mechanism that is mediated by the baroreceptors. ${ }^{1,4,6,16}$ Arterial blood pressure variability provides more direct assessment of autonomic nervous system 

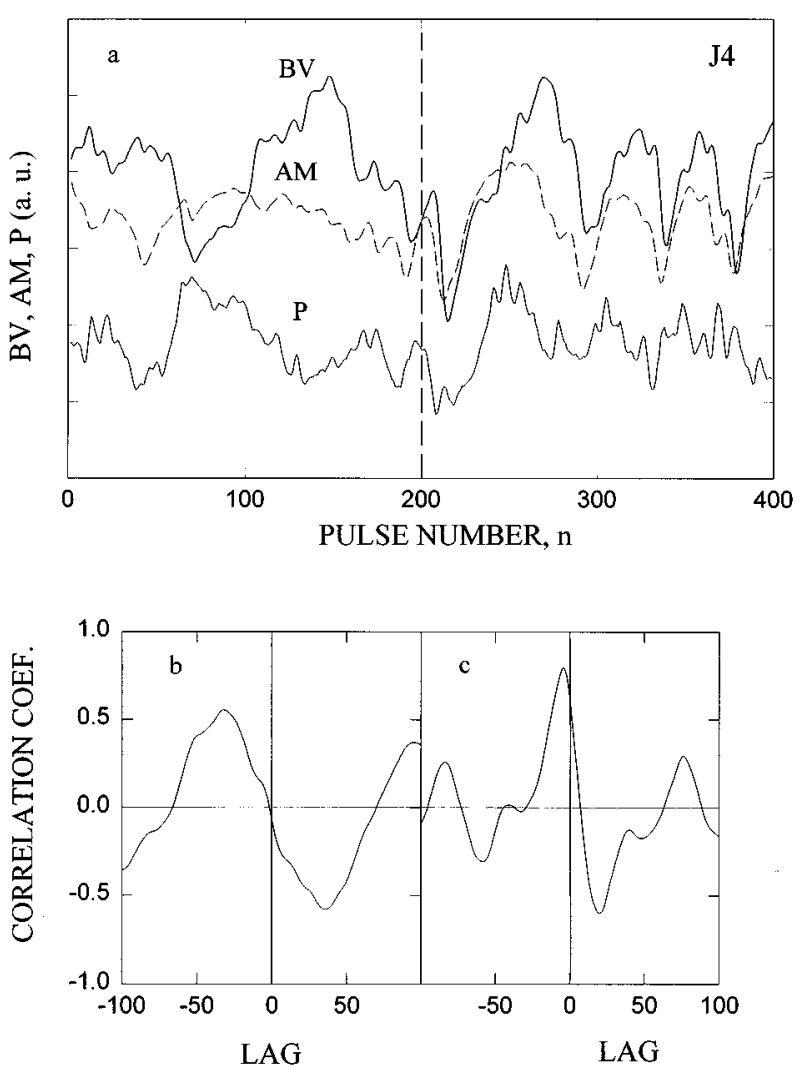

Fig. 6 (a) The curves of BV, $A M$, and $P$ as a function of pulse number after trend removal and smoothing for the same subject as in Fig. 3, in a different examination; (b) the cross-correlation function for the initial part of the BV and AM curves; (c) the crosscorrelation function for the final part of the BV and $A M$ curves.

control on the peripheral blood vessels than does HRV, but its measurement is performed either invasively, using an arterial catheter, or noninvasively but less accurately, using arterial compression. In both methods, the examination procedure may influence the arterial system, so that its fluctuations are not necessarily spontaneous.

In the current study, the PPG signal was obtained from the fingertips of ten subjects, and three parameters were derived from each pulse: $\mathrm{BV}$, which is directly related to the tissue blood volume; AM, which assesses the systolic blood volume pulse; and $\mathrm{P}$, the cardiac cycle period. All these parameters showed fluctuations in both low and high frequencies, but the relative intensity of the fluctuations in the two frequency ranges was different. The cardiac period curve fluctuated intensively in the high respiratory frequency, while the BV and AM curves showed higher fluctuations in the low-frequency range of a 30 to $50 \mathrm{~s}$ period. These results are in accord with those associated the low- and the high-frequency heart rate fluctuations to the sympathetic and the parasympathetic nervous systems, respectively. ${ }^{1,2,4}$ Since the parasympathetic nervous system mainly influences the heart, the variability in the high respiration frequency is expected to
Table 1 Maximum correlation coefficients and the corresponding lags for the cross-correlation functions of $B V$ versus $A M, B V$ versus $P$, and $A M$ versus $P$, and the values of the lag for maximum correlation.

\begin{tabular}{|c|c|c|c|c|c|c|c|}
\hline \multirow{2}{*}{$\begin{array}{l}\text { Subject } \\
\text { No. }\end{array}$} & \multicolumn{3}{|c|}{ BV vs. AM } & \multicolumn{2}{|c|}{ BV vs. $P$} & \multicolumn{2}{|c|}{ AM vs. $P$} \\
\hline & Lag & Slope & $\mathrm{R}$ & Lag & $\mathrm{R}$ & Lag & $\mathrm{R}$ \\
\hline 1 & 2 & 3.49 & 0.98 & 0 & -0.44 & -2 & -0.42 \\
\hline 2 & 4 & 4.68 & 0.91 & 6 & -0.39 & 2 & -0.25 \\
\hline 3 & 3 & 3.56 & 0.91 & 10 & 0.30 & 3 & 0.49 \\
\hline 4 & 6 & 4.17 & 0.89 & 12 & 0.55 & 6 & 0.65 \\
\hline 5 & 8 & 5.06 & 0.89 & 4 & -0.66 & -4 & -0.52 \\
\hline 6 & 2 & 3.14 & 0.87 & -6 & 0.60 & -5 & 0.60 \\
\hline 7 & 5 & 3.06 & 0.94 & 10 & 0.47 & 4 & 0.63 \\
\hline 8 & 4 & 4.27 & 0.96 & 7 & 0.65 & 2 & 0.70 \\
\hline 9 & 5 & 4.75 & 0.91 & 7 & 0.76 & 1 & 0.84 \\
\hline 10 & 5 & 3.98 & 0.89 & 3 & -0.13 & -6 & 0.15 \\
\hline Av. & 4.4 & 4.02 & 0.92 & 5.3 & 0.17 & 0.10 & 0.28 \\
\hline S.D. & 1.8 & 0.69 & 0.30 & 5.4 & 0.52 & 4.1 & 0.51 \\
\hline
\end{tabular}

have a strong effect on the P curve. The BV and AM values have pronounced fluctuations in the low frequency since both directly depend on the constriction and relaxation levels of the blood vessels, which are predominantly affected by the sympathetic nervous system. ${ }^{17}$

The decrease of AM, the systolic blood volume pulse, during periods of higher sympathetic nervous activity is due to the higher active constriction of the arteries and arterioles, which lowers their compliance. ${ }^{18-20}$ In most examinations, when a positive correlation between BV and AM was found, this higher arterial and arteriolar constriction also resulted in a reduction in tissue blood volume. This effect cannot be associated with the decrease in the arterial and arteriolar volume alone, which is the immediate result of the vessel constriction, since the BV fluctuations lag behind the AM fluctuations by 2 to 8 beats. The decrease in BV during the higher sympathetic activity should be attributed to the lower blood flow through the constricted arterioles, the resistance vessels, or the arteriovenous anastomoses (AVAs), resulting in a decrease in venous blood content. In a few examinations, however, the correlation between BV and AM changed to negative: the decrease in the systolic blood volume pulse was accompanied by an increase in the tissue blood volume. This inverse relationship between BV and AM can be interpreted by assuming a selective sympathetic constriction of the arterioles (the resis- 
tance vessels) and the AVAs, while the arteries are not actively constricted. The increased resistance of the constricted arterioles results in a reduction of blood flow through them and higher blood volume in the arteries which convey blood to the arterioles. If the blood volume increase in the arteries is higher than the decrease in venous blood content, the total blood volume in the tissue increases. The arterial blood volume increase results in simultaneous higher light absorption and higher values of BV with lower values of $A M$, due to the lower compliance ${ }^{12,21-23}$ of the passively distended arteries.

It should be noted that the negative correlation between BV and AM can be partly explained by the higher attenuation of the PPG signal for higher tissue blood volume. However, the small spontaneous changes in blood volume-typically 3 to $5 \%$ of the total blood volume-cannot account for the relatively high changes in AM-typically 20 to $30 \%$ of AM. There are also other phenomena that can contribute to the positive and negative correlations between BV and AM, and more studies should be performed in order to find out the contribution of each mechanism.

In previous studies the pulsatile or the average PPG signal was correlated with either the skin blood flow, measured by laser Doppler flowmetry ${ }^{8,9}$ or with arterial blood pressure. ${ }^{18}$ In another study, ${ }^{6}$ the air plethysmographic signal was correlated with arterial blood pressure. The study of the degree of correlation between the pulsatile PPG signal and its baseline has the advantage that both are measured at the same site.

The correlation between $\mathrm{BV}$ and $\mathrm{AM}$ versus $\mathrm{P}$ curves is even more complicated. The increased sympathetic nervous activity, which increases the constriction level of the arterioles, also has a direct effect on the heart, increasing the heart rate. ${ }^{16}$ Hence, for the majority of the examinations, the decrease in BV and AM was accompanied by a decrease in the PPG period P (i.e., the cardiac period), as can be seen in Fig. 4 and Table 1. Similar results were obtained by Burton, ${ }^{6}$ who found that a decrease in the plethysmographic signal was accompanied by a rapid increase in heart rate, and by Shimada and Marsh, ${ }^{24}$ who found a positive correlation between mean arterial blood pressure and heart rate in conscious dogs. It should be noted that a positive correlation between $\mathrm{AM}$ and $\mathrm{P}$ can be also attributed to the passive effect of decreased PPG amplitude and arterial pulse for decreased heart period. In our study, however, a negative correlation between $\mathrm{P}$ and $\mathrm{AM}$ or $\mathrm{BV}$ was found in some of the examinations. For the subjects who showed this result, a different physiological mechanism should be used to interpret the results. It seems that the increased constriction of the blood vessels due to the local regulation mechanism causes an increase of arterial blood pressure, which, via the negative feedback mechanism of the barore- ceptor reflex and the central autonomic nervous system, tends to decrease heart rate. ${ }^{1,4,15}$ A similar mechanism was suggested by Burton ${ }^{6}$ to explain the subsequent decrease of the heart rate after its initial increase during peripheral vascular constriction.

The regulation of the cardiovascular system by the autonomic nervous system is complex and composed of multiple components so that several physiological measurements are required in order to obtain adequate information on the different components of the two systems and their interrelationship. The measurement of the low-frequency fluctuations in tissue blood volume, systolic blood volume pulse and heart period, together with the correlations between them, seems to provide a potential tool for better understanding the sympathetic nervous control of the peripheral circulation and evaluation of its function. Though photoplethysmography is not appropriate for quantitative measurement of tissue blood flow or the systolic blood volume pulse in absolute terms, ${ }^{10}$ it can still be used as a simple and convenient means to assess the fluctuations of tissue blood volume, the systolic blood volume pulse, and the temporal relationship between them.

\section{Acknowledgment}

This research was supported by the E. W. Joseph Fund and by the Israeli Ministry of Science and Arts. Dr. Turivnenko and Dr. Babchenko were supported by the Israeli Ministy of Absorption.

\section{REFERENCES}

1. S. Akselrod, D. Gordon, J. B. Madwed, N. C. Snidman, D. C. Shannon, and R. J. Cohen, "Hemodynamic regulation: investigation by spectral analysis," Am. J. Physiol. 249, H867H875 (1985).

2. A. Malliani, M. Pagani, F. Lombardi, and S. Cerutti, "Cardiovascular neural regulation explored in the frequency domain," Circulation 84, 482-492 (1991).

3. M. Muzi and T. J. Ebert, "Quantification of heart rate variability with power spectrum analysis," Cur. Opin. Anaesthiol. 6, 3-17 (1993).

4. C. M. A. van Ravenswaaij-Arts, L. A. A. Kollee, J. C. W. Hopman, G. B. A. Stoelinga, and H. P. van Geijn, "Heart rate variability," Ann. Intern. Med. 118, 436-447 (1993).

5. R. W. de Boer, J. M. Karemaker, and J. Strackee, "Hemodynamic fluctuations and baroreflex sensitivity in humans: A beat-to-beat model,"' Am. J. Physiol. 253, 680-689 (1987).

6. A. C. Burton, "The range and variability of the blood flow in the human fingers and the vasomotor regulation by body temperature," Am. J. Physiol. 127, 437-453 (1939).

7. K. Lossius, M. Eriksen, and L. Walloe, "Fluctuations in blood flow to acral skin in humans: Connection with heart rate and blood pressure variability," J. Physiol. 460, 641-655 (1993).

8. H. Schmid-Schonbein, S. Ziege, W. Rutten, and H. Heidtmann, "Active and passive modulation of cutaneous red cell flux as measured by laser Doppler anemometry," VASA Supp. 34, 38-47 (1992).

9. N. E. Almond, D. P. Jones, and E. D. Cooke, "High quality photoplethysmograph signals from a laser Doppler flowmeter: Preliminary studies of two simultaneous outputs from the finger," J. Biomed. Eng. 10: 458-462 (1988).

10. A. Fronek, Noninvasive Diagnosis in Vascular Disease, pp. 22 27, McGraw-Hill, New York (1989). 
11. T. M. R. Shankar and J. G. Webster, "Contribution of different sized vessels in the extremities to the arterial pulse waveform as recorded by electrical impedance and volume plethysmography," Med. Biol. Eng. Comput. 23, 155-164 (1985).

12. M. Nitzan, Y. Mahler, S. Yaffe, R. Marziano, M. Bochar, and R. Chissin, "ECG-gated radionuclide plethysmography--a method for the assessment of pulmonary systolic blood volume increase," Clin. Phys. Physiol. Measurement 13, 21-28 (1992).

13. M. Nitzan, H. de Boer, S. Turivnenko, A. Babchenko, and D. Sapoznikov, "Spontaneous oscillations in the peripheral circulation system as measured by photoplethysmography," in Proc. Europto Conf. for Biomedical Optics, SPIE, Washington, Vol. 2328, pp. 188-195 (1994).

14. M. Nitzan, H. de Boer, S. Turivnenko, A. Babchenko, and D. Sapoznikov, "Power spectrum analysis of the spontaneous flucations in the photoplethysmographic signal," J. Basic Clin. Physiol. Pharmacol. 5, 269-276 (1994).

15. D. Sapoznikov, M. H. Luria, Y. Mahler, and M. S. Gotsman, "Computer processing of artefact and arrhythmias in heart rate variability analysis," Comp. Methods Prog. Biomed. 39, 75-84 (1992).

16. W. Craelius, V. K. H. Chen, M. Restiva, and N. El-Sherif, "Rhythm analysis of arterial blood pressure," IEEE Tr. BME 33, 1166-1172 (1986)
17. A. C. Guyton, Textbook of Medical Physiology, 7th ed., pp. 345, 692, W. B. Saunders, Philadephia, 1982.

18. K. H. Wesseling, J. J. Settels, G. M. A. Van der Hoeven, J. A. Nijboer, M. W. A. Butijn, and J. C. Dorlas, "Effects of peripheral vasoconstriction on the measurement of blood pressure in the finger," Cardiovasc. Res. 19, 139-145 (1985).

19. K. Yamakoshi and A. Kamiya, "Noninvasive and simultaneous cardiovascular measurements using plethysmography techniques," Frontiers Med. Biol. Eng. 2, 249-276 (1990).

20. M. Y. Jaffrin and C. Vanhoutte, "Quantitative interpretation of arterial impedance plethysmographic signals," Med. Biol. Eng. Comput. 17, 2-10 (1979).

21. M. E. Safar, B. I. Levy, S. Laurent, and G. M. London, "Hypertension and the arterial system: Clinical and therapeutic aspects," J. Hypertension 8, S113-S119 (1990).

22. F. Perret, V. Mooser, D. Havoz, Y. Tardy, J. J. Meister, J. D. Etienne, P. A. Farine, A. Marazzi, M. Burnier, J. Nussberger, B. Waeber, and H. R. Brunner, "Evaluation of arterial compliance-pressure curves; effect of antihypertensive drugs," J. Hypertension 18 (Suppl II):II-77-II-83 (1991).

23. D. S. Berger and J. K. J. Li, "Temporal relationship between left ventricular and arterial system elastances," IEEE Trans. Biomed. Eng. 39, 404-410 (1992).

24. S. G. Shimada and D. J. Marsh, "Oscillations in mean arterial blood pressure in conscious dogs," Circ. Res. 44, 692-700 (1979). 